\title{
Improvement of Imaging Performance with a New ASCOR Probe-Corrector in a 200 kV JEM-ARM200CF
}

\author{
M. Watanabe ${ }^{1}$, T. Nakamura ${ }^{2}$, T. Ishikawa ${ }^{3}$ \\ 1. Dept of Materials Science and Engineering, Lehigh University, Bethlehem. PA 18015. \\ 2. JEOL USA Inc.. Peabody, MA 01960. \\ 3. JEOL Ltd., 3-1-2 Musashino, Akishima, Tokyo, 196-8558, Japan.
}

Imaging and chemical analysis at atomic-level spatial resolution with single-atom detection sensitivity is one of the ultimate goals in materials characterization. It is no longer dream to perform such characterization in atomic scale when aberration-corrected scanning transmission electron microscopes (STEMs) with electron energy-loss spectrometry (EELS) and X-ray energy dispersive spectrometry (XEDS) are employed because long-term instrumental and environmental stabilities required for atomic-resolution characterization have been improved significantly. The JEOL JEM-ARM200CF is one of latest aberration-corrected STEMs designed for atomic-resolution imaging and analysis and has been intentionally used at Lehigh. For further improvement in imaging and analytical performance, this instrument was upgraded by swapping the existing CEOS CESCOR corrector with the latest CEOS ASCOR corrector, which is designed to perform better tuning for $3^{\text {rd }}-5^{\text {th }}$ order aberrations. In this study, improved imaging performance after installation of the ASCOR to JEM-ARM200CF is introduced.

The latest-generation of aberration-corrector design can significantly improve corrections of higher-order (i.e. $3^{\text {rd }}$ and $4^{\text {th }}$ order) geometric aberrations. Fig. 1 shows the $59 \%$ probe diameter simulated at several different conditions, plotted against the probe forming angle [1]. Once the $3^{\text {rd }}$ and $4^{\text {th }}$ order geometrical aberrations are tuned (the residual aberrations are a $5^{\text {th }}$-order spherical aberration and six-fold astigmatism), the aberration-free angle reaches at least $40 \mathrm{mrad}$, as shown in the geometrical probe size. However, due to the chromatic aberration caused by the energy-spread of the electron source, the angle is strictly limited to $\sim 25 \mathrm{mrad}$ in a Schottky source instrument (suitable angular ranges for the conventional correctors). Conversely, in a cold-FEG instrument, the angle can be as high as $35 \mathrm{mrad}$ in reality because of the smaller energy spread. Therefore, the tuning capability of higher-order aberrations in the ASCOR corrector is essential to fully utilize the potential of cold-FEG instrument.

Figure 2 shows two Ronchigrams obtained after tuning at 200 and $60 \mathrm{kV}$, respectively. At both accelerating voltages, the aberration-free semi-angles are expanded to over $60 \mathrm{mrad}$. It should be noted that the $5^{\text {th }}$-order spherical aberration coefficient is below $50 \mu \mathrm{m}$ after the corrector tuning and other higher-order aberrations are reduced accordingly. Figure 3 shows a high-angle annular dark-field (HAADF) STEM image of [211]-projected GaN taken at $200 \mathrm{kV}$. In Fig. 3, a fast Fourier transformed (FFT) diffractogram is also inserted, which indicates reflections at $63 \mathrm{pm}$. In addition, a HAADF-STEM image of [112]-projected Ge recorded at $60 \mathrm{kV}$ is shown in Fig. 4 with its FFT diffractogram exhibiting $82 \mathrm{pm}$ reflections.

The latest ASCOR probe corrector provides better tuning performance for $3^{\text {rd }}-5^{\text {th }}$ order aberrations, which offers expansion of aberration-free angular ranges, so that lateral resolution reaches to $\sim 0.6 \AA$ at 
$200 \mathrm{kV}$ and to below $1 \AA$ at $60 \mathrm{kV}$ in the JEM-ARM200CF. The expansion of aberration-free angular range also provides improvements for depth resolution and sensitivity in through-focal STEM imaging. The analytical performance i.e. resolution and sensitivity, can also be improved by the larger aberration-free angular range. In the presentation, the improved analytical performance will also be introduced. [2]

\section{References:}

[1] M. Watanabe, in Scanning Transmission Electron Microscopy Eds. by S.J. Pennycook and P.D. Nellist, Springer NY, (2011), p.291.

[2] The author (MW) wishes to acknowledge financial support from the NSF through grants DMR-0804528 and DMR-1040229.

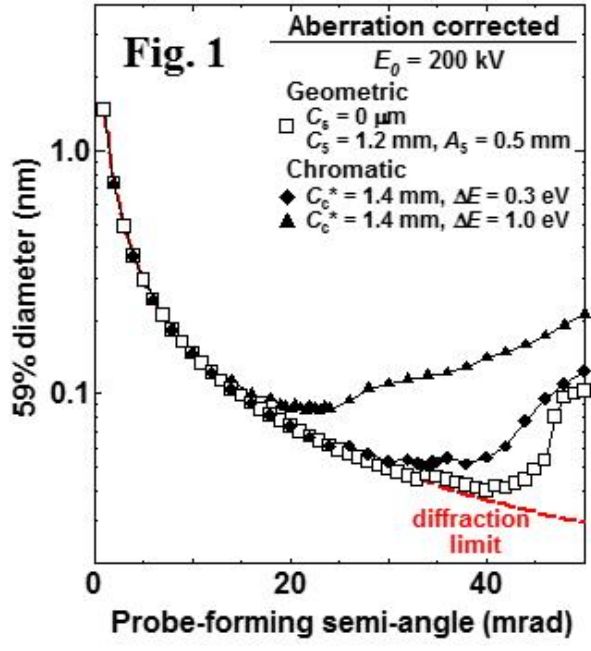

Fig. 3

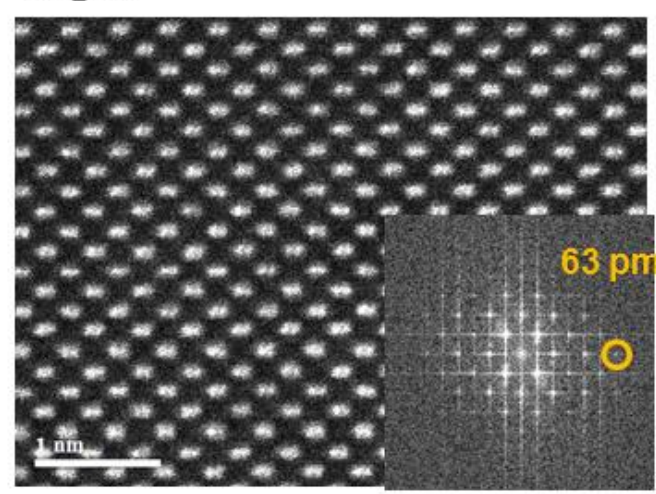

Fig. 2
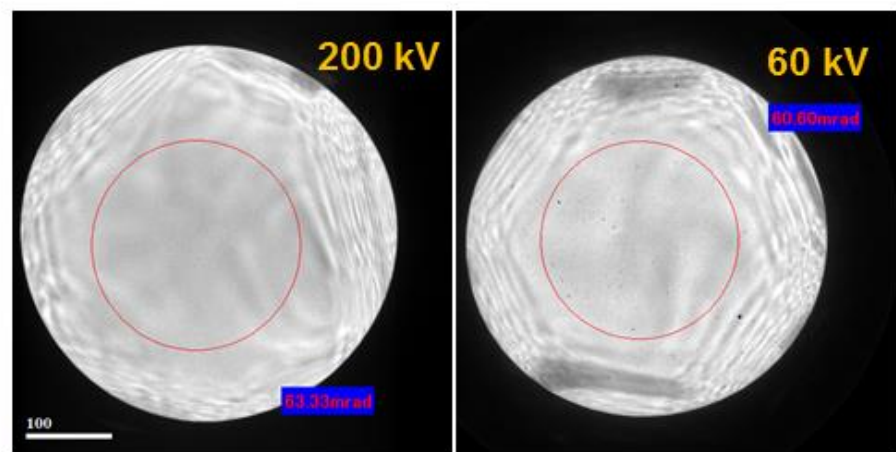

Fig. 4

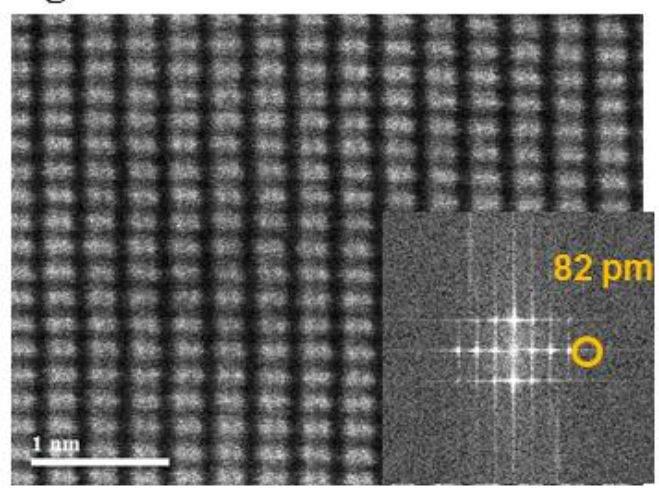

Figure 1. The 59\% probe diameters for Schottky FEG and cold FEG aberration-corrected JEM-ARM200CF, plotted against the probe-forming semi-angle $\square$.

Figure 2. Romchigrams after the ASCOR corrector tuning: (left) at $200 \mathrm{kV}$ and (right) at $80 \mathrm{kV}$.

Figure 3. A HAADF-STEM image of [211]-projected GaN taken at $200 \mathrm{kV}$, with its FFT diffractogram.

Figure 4. A HAADF-STEM image of [112]-projected Ge recorded at $60 \mathrm{kV}$, with its FFT diffractogram. 